patients might have received a favourable bias as they tended to have aneurysm surgery earlier, and there were no postoperative deaths or rebleeding.

The restriction of my study to patients who had survived the presenting haemorrhage in a good condition was deliberate. In the past confusion has arisen because of failure to distinguish between the many deaths from effects of the first bleed, often days or weeks after reaching hospital, and those due to recurrent haemorrhage. Obviously, antifibrinolytic treatment (or surgery) can only improve mortality in the latter group. Inclusion of obtunded patients in a trial will lead to inclusion of many deaths from the first bleed, as in the study of van Rossum et al, where 18 patients died from the primary bleed as against only seven from rebleeding. I have already suggested in my paper that antifibrinolytic treatment might be positively harmful in patients in poor condition.

The trial of van Rossum et al was small. Of 51 patients admitted, only 24 proved to have aneurysms on angiography. The discrepancy with my findings may be due partly to delay in starting treatment and partly to the fact that their patients received only $4 \mathrm{~g}$ of tranexamic acid a day and for only 10 days. The fibrinolytic activity induced in the nervous system after a subarachnoid haemorrhage may last for much longer than this as shown in my study.

I feel that the comments of Drs van Rossum and Wintzen on the existing literature are hardly fair. Earlier studies have had a limited follow-up as the antifibrinolytic treatment was used merely as a prelude to surgery. The study referred to in my article as a "personal communication" from Dr Fodstad has now been published. ${ }^{2}$ It provides further evidence for the apparent efficacy of antifibrinolytic treatment.

R S MAURICE-WILLIAMS Regional Neurosurgical Unit, Brook General Hospital, London SE18

1 van Rossum, J, et al, Annals of Neurology, 1977, 2, 242

\section{Cervical presentation of rectal carcinoma}

SIR,-We were very interested to read the case report by Dr M J Sworn and others (8 July, p 98) on "Rectal carcinoma presenting as massive metastatic involvement of foot bones." The authors commented that this was the first report of such massive metastatic disease of the foot. We would like to present another such case with the primary tumour being in the cervix.

A 52-year-old woman presented with occasional blood-stained vaginal discharge and after a normal clinical examination underwent dilatation and curettage. Histological examination of the curettings showed fragments of moderately differentiated squamous carcinoma. A further punch biopsy of the posterior vaginal wall showed infiltration by moderately differentiated squamous carcinoma obviously derived from the cervix. Treatment consisted in radical radiotherapy using two insertions of caesium and external megavoltage $x$-ray treatment.

Over a year later she was referred for review on account of a two-month history of increasing pain in her right foot culminating in inability to bear weight. A radiograph (see figure) showed extensive metastatic disease affecting

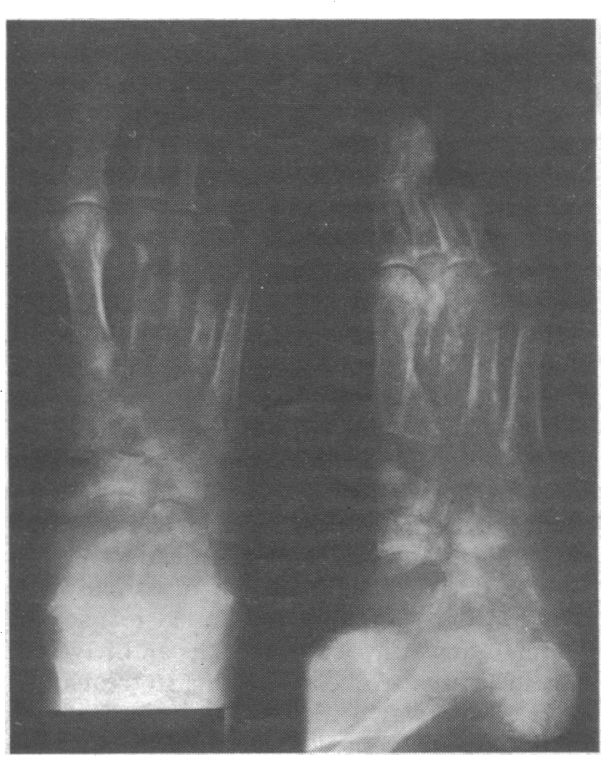

Extensive metastatic disease of right foot.

the whole foot, including the calcaneum, talus, navicular, cuboid, the three cuneiform bones, the five metatarsals, and with probably further deposits in the phalanges. A bone scan showed other metastatic areas of skeletal involvement -namely, the ipsilateral tibia and upper femur, pelvis, and lumbar spine. A biopsy specimen taken from the mid-tarsus showed invasive moderately to poorly differentiated squamous carcinoma fully consistent with the original tumour of cervix. The patient has recently had palliative radiotherapy to control her symptoms.

T I S BROWN

Princess Margaret Rose Orthopaedic

G L RITCHIE

Hospital,

Edinburgh EH10 7ED

\section{Thyroid extract}

SIR,-My friend Dr P B S Fowler (26 August, p 636) has, I think, misunderstood the letter from Dr W van't Hoff and his colleagues (15 July, p 200). The danger to which they refer is a negative one. The potency of thyroid extract is very variable and I have on several occasions seen a hypothyroid patient successfully treated with the extract who has suddenly become myxoedematous. The onset of myxoedema is often so insidious that the risk of coronary heart disease to which Dr Fowler has so often drawn our attention must in such patients be increased.

RAYMOND GREENE

London W 1

\section{Whooping-cough fatality rate}

SIR,-Dr Dick (19 August, p 562) suggests that the fatality rate for pertussis may have diminished. He uses as his denominator notifications.

General practitioners are rightly selective in the way they choose to respond to the notification of infectious disease, and notifications are a notoriously unreliable indicator of the incidence of common diseases. The present interest in the incidence of pertussis is likely to increase the proportion of cases notified. As fatalities are always recorded an increase in the proportion of cases notific d will diminish the case fatality rate.

Until we have reliable measures of incidence we can make no valid statements regarding case fatality.

JAMES MCCORMICK

University Department of Community

Health,

St James's Hospital,
Dublin

\section{Paracetamol poisoning}

SIR,-Dr T J Meredith and others (12 August, p 478) quote from the Mortality Statistics $^{1}$ that in England and Wales in 1975 there were 105 deaths due to paracetamol and a further 107 due to paracetamol in combination with other drugs. It could be inferred from this statement that all these deaths were the direct result of paracetamol overdosage. In table 10 of the Mortality Statistics it is noted that inclusion in the list does not necessarily imply that a substance was the cause of death even where solely involved. With this in mind, we have carried out a study which indicates that the recorded number of paracetamol deaths considerably exceeds the actual number which can be directly attributed to paracetamol.

The pathology of paracetamol overdosage is now clearly defined with the clinical course being consequent on the extent of any hepatic damage. Death due to paracetamol overdosage is primarily caused by hepatic failure and occurs some days after ingestion of the overdose. ${ }^{2}$ Our study included a consideration of additional data made available to us by courtesy of the Office of Population Censuses and Surveys. These showed that evidence of hepatic necrosis was reported in only 50 out of the 105 deaths attributed to paracetamol alone. Additional information was obtained in 50 of the remaining 55 cases by courtesy of HM Coroners under whose jurisdiction the inquests were held. From this it was found that in 12 cases there was evidence of hepatic necrosis resulting in death, and in one instance hepatic necrosis was present but was not the immediate cause of death (peritonitis from perforated peptic ulcer). In the remaining 37 cases, however, death was sudden, and at necropsy the liver was regarded as normal. Twenty-five of these individuals were known to have ingested, in addition to paracetamol, other drugs, which were mainly those with a respiratory depressant action, particularly dextropropoxyphene. There is no evidence that the paracetamol contributed in any way to these deaths. The Office of Population Censuses and Surveys also provided us with additional data on a further 112 cases where paracetamol was listed together with other substances. As 84 of these people died before being admitted to hospital we consider it unlikely that paracetamol contributed to their death. Of the 28 who died in hospital, there were only two reported cases of hepatic necrosis while 20 died from the results of overdosage of respiratory depressant drugs taken with paracetamol. In the remaining six cases the possibility that paracetamol was the cause of death could not be excluded.

We therefore estimate that the total number of deaths definitely attributable to paracetamol in 1975 in England and Wales amounted to 64. In 37 cases listed as due to paracetamol alone there was no evidence of paracetamol toxicity, and in 104 cases where paracetamol was taken in combination with other drugs it is unlikely 